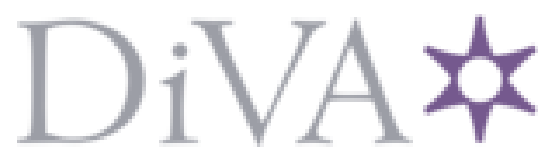

http://www.diva-portal.org

\title{
Postprint
}

This is the accepted version of a paper presented at IEEE International Symposium on Communications, Control, and Signal Processing,May 21-24, 2014.

Citation for the original published paper:

Alesii, R., Congiu, R., Santucci, F., Di Marco, P., Fischione, C. (2014)

Architectures and protocols for fast identification in large-scale RFID systems.

In:

N.B. When citing this work, cite the original published paper.

Permanent link to this version:

http://urn.kb.se/resolve?urn=urn:nbn:se:kth:diva-141179 


\section{Architectures and protocols for fast identification in large-scale RFID systems}

\author{
R. Alesii, R. Congiu, F. Santucci, \\ Centre of Excellence DEWS \\ University of L'Aquila, Italy \\ roberto.alesii@univaq.it, robcong.rc@gmail.com \\ fortunato.santucci@univaq.it
}

\author{
P. Di Marco, C. Fischione. \\ ACCESS Linnaeus Centre \\ Royal Institute of Technology, Sweden \\ pidmakth.se, carlofi@kth.se
}

\begin{abstract}
Passive tags based on backscattered signals yield low energy consumption for large-scale applications of RFIDs. In this paper, system architectures and protocol enhancements for fast identifications in ISO/IEC 18000-6C systems that integrate UWB technology are investigated. The anti-collision protocol is studied by considering various tag populations. A novel algorithm is proposed to adapt the UHF air interface parameters with the use of UWB ranging information. The results show that the proposed algorithm yields up to $25 \%$ potential performance improvement compared to the ISO/IEC 18000-6C standard.
\end{abstract}

Index Terms - RFID, Medium Access Control, Ultra-wide band

\section{INTRODUCTION}

Radio Frequency IDentification (RFID) denotes a dedicated short range communication technology for automated object identification, with applications spanning from inventory control and object tracking to electronic tolling and cardreading. Compared to optical bar-code scanning technology, RFID is faster and more efficient for automatically collecting information [1]. The main components of an RFID system are a reader (interrogator) and a tag (transponder). The reader interrogates a group of tags in its vicinity to read their IDs and exchange information about related objects. Active tags contain an embedded power source and computation capabilities, while passive tags must harvest energy from the reader signals. The available energy for passive tags is scarce and often intermitting, therefore fast identification procedures are necessary.

The integration of backscattering ultra-wide band (UWB) technology and RFID tags is a promising step towards the definition of green RFID systems, where an emerging requirement consists in reducing energy consumption and environmental impact (e.g. through the use of recyclable materials) [2]. Moreover, the capability of localizing and tracking passive (or semi-passive) devices through UWB signals is a key feature for enabling support of a rich applications set and improving performance and efficiency of existing systems [3].

The ISO/IEC 18000-6 [4] is the reference standard for RFID systems operating in the $860 \mathrm{MHz}-960 \mathrm{MHz}$ UHF

The authors acknowledge the support of the Swedish Research Council, the EU project Hycon2, and the MIUR Italian PRIN project GRETA under Grant 2010WHY5PR. frequency range. It adopts and enhances the specifications of the EPCglobal protocol Class-1 Generation-2, which is used by most of the RFID manufacturers [5]. In our study, we focus on the Type C modality, which employs a framed slotted ALOHA collision arbitration with dynamic frame adaptation.

The objective of this paper is performance analysis of the medium access control (MAC) of the ISO/IEC 180006 standard. The presence of UHF backscattering signals is peculiar with respect to other wireless communication technologies. The effects of air interface parameters on the system performance in terms of success rate and identification speed are investigated. Furthermore, we exploit ranging/localization capabilities of tags that are ensured by the UWB component to propose an enhanced MAC protocol that operates with wireless coverage awareness.

The remainder of the paper is organized as follows. In Sect. II, we survey the existing literature. The system architecture is illustrated in Sect. III. We describe the ISO/IEC 180006C protocol in Sect. IV and develop performance indicators in Sect. V. The proposed mechanism is illustrated in Sect. VI and compared to the standard anti-collision protocol. Finally, Sect. VII concludes the paper and prospects our future work.

\section{RELATED WORK}

There is a lively research interest on MAC solutions for RFID systems based on the ISO/IEC 18000-6 standard (i.e, [6] - [14]). In [6], the authors propose a tree structured collision resolution scheme. The energy performance of tree structured protocols is studied in [7]. A token MAC protocol is proposed in [9], introducing tag fairness as a performance metric. In [10], the performance of the ISO/IEC 18000-6C is characterized by a Markov chain model. The performance metrics considered therein are the reliability in terms of the query success rate and the latency in terms of tag identification speed. A study of the energy performance is proposed in [11] and it includes energy harvesting of the passive tags. In [12], mechanisms for collision recovery that assume multi-packet reception are studied and compared to real measurements. In [13], the optimal contention window is derived according to estimations of the tag population. In [14], an adaptive algorithm for the contention window is proposed to maximize the number of reads per second. The presence of multiple 


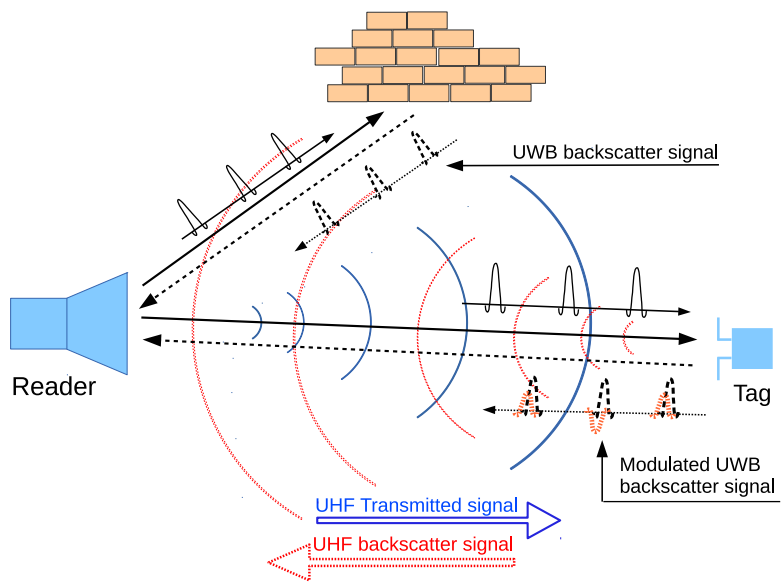

Fig. 1. System architecture and propagation scenario.

access interference and the impact on the MAC performance has been studied in [15]. A key factor in the aforementioned protocols is the dependence of the performance on the tag population size and the ability to estimate the number of unidentified tags.

The adoption of UWB technology in RFID systems has been advocated in recent literature to support accurate real-time localization, security, and large-scale system management [2], [16]. However, the possible integration with the existing standard UHF RFID technology has not been investigated yet and is one of the targets of GRETA project.

\section{System ARCHITECTURES FOR LARGE-SCALE RFID SYSTEMS}

We consider a fully compliant ISO/IEC 18000-6C RFID architecture with further provision of UWB technology [17]. In particular, the UHF ISO/IEC 18000-6C protocol is used for synchronization and identification, while the UWB technology is used for ranging and localization. It is worthwhile mentioning that this integrated UHF/UWB architecture is one of the options devised in the GRETA project in order to progress towards zero-power RFID systems that are nevertheless able to support accurate localization and tracking of entities. While the overall project target encompasses many challenging topics (from paper-based substrates for RFID tags to cooperative algorithms for tracking), in the specific context of this article we are interested on exploiting the integrated wireless architecture in order to propose an enhanced MAC protocol that relies on the accurate ranging capabilities provided by the UWB component.

The system architecture and a sketch of propagation scenario are illustrated in Fig. 1:

- In the UHF domain, the tag collects power from the RF signal transmitted by the RFID reader and sends data back by switching its input impedance between two states, thus modulating the backscattered UHF signal.

- In the UWB domain, the already energized tag may transmit back by modulating a sequence of backscattered UWB pulses.
Various solutions for UWB detection and clutter suppression are discussed in [18]; however, this topic is out of the scope of this paper.

\section{A. Ranging and localization}

Let us consider a reader that interrogates a set of tags in its communication range by transmitting an UWB signal with a specific spreading code. A tag replies by modulating the signal backscattered from its antenna. By measuring the time of arrival (ToA) of the backscattered signal, the reader is able to estimate the distance (ranging operation) and then provide an input to one or more algorithms for estimating the position of the tag and/or reader. When dealing with the successive step of localization, interplay/cooperation among more (fixed) readers is required to localize/track sets of nomadic tags; in the case of nomadic readers, cooperation among readers may be required to first estimate their absolute or relative position. Furthermore, in a different application setting, a mobile reader may estimate its position by relying on ranging with a set of fixed (anchor) tags.

\section{B. Clock synchronization}

Accomplishing frequency and time synchronization is of particular relevance in the depicted system architecture. We then envisage cooperating UWB and UHF systems for clock synchronization.

The internal clock of the tag might differ from the reader clock. By keeping a simple tag implementation, a free running oscillator could be integrated in the tag. However, clock drifts and code offsets become critical problems for the complexity of detection algorithms on the reader side. As a matter of fact, before decoding the received data transmitter frequency and timing must be synchronized. This is performed in ISO 18000-6 devices with standard mechanisms [10], [19] that are briefly described in the following.

Every reader message form must begin with a preamble or a frame synchronization. The reader transmits a continuous wave (CW) to the tag. A Pulse Interval Encoding (PIE) is used to transmit data from the reader to the tag. This encoding method implicitly includes the clock to easily detect the bit (or symbol) boundaries at the tag with a low complexity hardware. The first step in the demodulation process at the tag is to suppress the carrier by detecting the envelope. The envelope is then passed through a trigger which detects the rising and falling edges of the envelope and cleans it from the noise. The trigger output is inverted to get the clock, which is used reset the subsequent integrator and in all digital processing of the tag. The decoded data is passed through the digital part in-parallel with the clock if it needs to be processed.

\section{ISO/IEC 18000-6C MAC}

According to the specifications of ISO/IEC 18000-6 MAC, an interrogation round is defined as a sequence of consecutive commands and responses that are issued by the reader and replied back by the tag in order to transmit the tag ID to the reader. A sequence of consecutive interrogation rounds forms 
an interrogation session. At the beginning of the session, the reader sends a Query command with an integer parameter $Q$, asking every tag to generate a 16-bits random number (RN16) uniformly and to mask $Q$ bits of the generated RN16 to determine their slot counter. At each round, the reader sends QueryRep commands in response of which tags decrement the slot counter. Tags respond with their RN16 when their counter counts down to 0 , forming a framed slotted ALOHA multi-access system over a duration of $2^{Q}$ slots.

Every round starts with a Query/QueryRep/QueryAdj command which asks the tag with zero slot counter to communicate its RN16. Following the Query/QueryRep command, the reader transmits an unmodulated carrier, and listens to the tag replies. Assuming that a single tag replies, the reader acknowledges by sending an ACK that includes the received RN16. Every tag decodes the ACK; if the included RN16 matches its own RN16 at a tag, it responds by transmitting its packet (denominated EPC message). After sending the packet, the tag will not respond to Query/QueryRep/QueryAdj messages in the same session. If the packet is not detected, the reader issues a NACK to notify the tag that remains active in the arbitration.

A Query $A d j$ command is issued by the reader to instruct all tags to adjust the value of $Q$ and re-select their slot number based on the new $Q$ value according to an adaptive $Q$ algorithm, which we describe next.

\section{A. Adaptive $Q$-algorithm}

The standard $Q$ algorithm in ISO/IEC 18000-6C is based on an additive increase/decrease mechanism, such that the value of $Q$ is either incremented when multiple collisions are detected or decremented after consecutive idle rounds. The mechanism is illustrated in Fig. 2.

The increment/decrement rate of $Q$ is specified by a parameter $c$. A variable $Q_{f p}$ is defined such that at each collision, the value of $Q_{f p}$ is incremented by $c$. If $Q_{f p}$ exceeds a maximum value $Q_{\max }$, it is set at $Q_{\max }$. Similarly, the value of $Q_{f p}$ is decremented by $c$ at each idle round. If $Q_{f p}$ is lower than a minimum value $Q_{\min }$, it is set at $Q_{\min }$. The value of $Q$ is selected as the nearest integer to $Q_{f p}$, i.e., $Q=\operatorname{round}\left(Q_{f p}\right)$. The value of $Q$ is kept unchanged whenever the interrogation is successful.

The ISO/IEC 18000-6 Type C standard [4] sets an initial value of $Q$ as $Q_{0}=4$, while $c$ is in the range $0.1 \leq c \leq 0.5$. Default parameters are $Q_{\min }=0$ and $Q_{\max }=15$.

\section{Performance AnAlysis of the ISO/IEC 18000-6C}

We utilize two indicators to evaluate the performance of the MAC as follows [10].

- Query Success Rate (QSR): it defines the average rate at which query commands successfully identify a tag: in other words, it is the average probability of identifying a tag in a query round.

- Tag Interrogation Speed (TIS): it defines the average rate at which tags are successfully identified. It accounts for the different duration of idle rounds, collided rounds, and successful rounds.

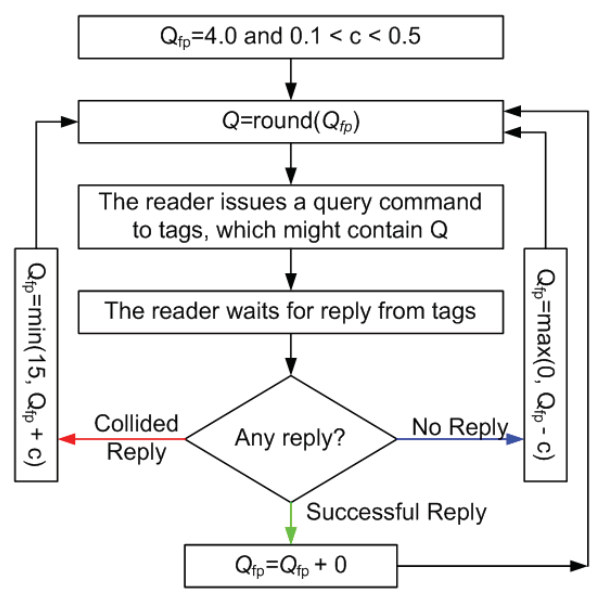

Fig. 2. $Q$-adaptation algorithm in the ISO/IEC 18000-6C MAC.

Both the QSR and the TIS depend on protocol specifications as well as the number of tags, the distance between the tag and the reader, environment, etc. In this paper we assume static channel conditions and zero bit-error rate. Moreover, as we are interested on assessing basic performance of the MAC protocol, we here neglect the effects of antenna polarization on determining the communication range of the reader.

\section{A. QSR analysis}

Recalling the basic MAC mechanism described in Sect. IV, a query is successful if there is only one tag selecting the current round. In the classic slotted ALOHA, by considering $N$ tags to be identified with a probability $p$ of selecting a specific round, the probability of successful interrogation can be written as

$$
P_{s}=N p(1-p)^{N-1},
$$

which is maximized for $p=p^{\star}=1 / N$ [20].

In the ISO/IEC 18000-6C MAC, the backoff increase is exponential and the probability of selecting a specific round is $p=1 / 2^{Q}$, where $Q_{\min } \leq Q \leq Q_{\max }$, and $Q \in \mathbb{Z}$. The value of $Q$ that maximizes the probability of successful interrogation is then the solution of the following optimization problem:

$$
\begin{array}{ll}
\underset{Q}{\operatorname{maximize}} & N \frac{1}{2^{Q}}\left(1-\frac{1}{2^{Q}}\right)^{N-1} \\
\text { subject to } & Q_{\min } \leq Q \leq Q_{\max }, \quad Q \in \mathbb{Z}
\end{array}
$$

Since the number of tags is not known in advance at the reader and it decreases with time as tags are identified, the adaptive Q-algorithm is used to select $Q$ during an interrogation session. The QSR is then the probability of successful interrogation averaged over the interrogation session.

Considering the processes $n_{t}$ and $q_{t}$ that denote respectively the number of unidentified tags and the value of $Q$ at the round $t$, the interrogation session is described as a discrete time Markov chain (see [10], [15]) and the QSR is

$$
\mathrm{QSR}=\frac{1}{N} \sum_{t=1}^{\infty} n_{t} \frac{1}{2^{q_{t}}}\left(1-\frac{1}{2^{q_{t}}}\right)^{n_{t}-1} .
$$


The pair $\left(n_{t}, q_{t}\right)$ represents the state of a two-dimensional discrete time Markov chain [10], [15].

\section{B. TIS analysis}

Timing is introduced by considering three terms: $T_{\text {succ }}$, $T_{\text {coll }}$, and $T_{\text {idle }}$, defined as the time duration of a successful identification, the time duration for a collided reply, and the time duration of an idle round, respectively:

$$
\begin{aligned}
T_{\text {succ }} & =2\left(T_{1}+T_{2}\right)+T_{q}+T_{r n}+T_{\mathrm{ack}}+T_{\mathrm{epc}}, \\
T_{\text {coll }} & =\left(T_{1}+T_{2}\right)+T_{q}+T_{r n}, \\
T_{\text {idle }} & =\left(T_{1}+T_{3}\right)+T_{q},
\end{aligned}
$$

where $T_{q}$ is the query transmission time , $T_{r n}$ is the transmission time of the RN16 to the reader, $T_{\text {ack }}$ is the ACK transmission time, $T_{\text {epc }}$ is the transmission time of the EPC message, $T_{1}$ is the tag response time after reader transmissions, $T_{2}$ is the reader response time, and $T_{3}$ is the time a reader waits, after tag response, before it issues another command.

Since the three types of query commands have different length, we consider $T_{q 1}$ for Query, $T_{q 2}$ for Query $A d j$, and $T_{q 3}$ for QueryRep. Similarly, there are three possible values for $T_{\text {succ }}, T_{\text {coll }}$, and $T_{\text {idle }}$.

The TIS is then obtained as

$$
\mathrm{TIS}=\frac{N}{N \bar{T}_{\text {succ }}+N_{c} \bar{T}_{\text {coll }}+N_{i} \bar{T}_{\text {idle }}},
$$

where $N_{c}$ and $N_{i}$ are the number of collided rounds and idle rounds, respectively, $\bar{T}_{\text {succ }}, \bar{T}_{\text {coll }}$, and $\bar{T}_{\text {idle }}$ are the average time durations for success, collision, and idle round, with respect to the number of Query/QueryAdj/QueryRep commands.

\section{Considerations}

Given default air interface parameters in [4], the following relation holds $T_{\text {idle }} \leq T_{\text {coll }} \leq T_{\text {succ }}$. QSR and TIS can be then both maximized by optimizing the probability of successful interrogation in each round. Moreover, given a fixed QSR target, the TIS can be improved by considering policies that penalize collision events more than idle rounds.

In Fig. 3, we report the theoretical probability of successful interrogation vs. the current number of unidentified tags. We compare the classic slotted ALOHA and the ISO/IEC 180006C MAC. The probability of successful interrogation of both systems quickly decreases as the number of unidentified tags increases, with theoretical bound at $P_{s} \approx 0,367$ for $N \rightarrow \infty$. For ISO/IEC 18000-6C MAC, local maxima are achieved for $N=2^{Q}, Q \in \mathbb{Z}$. The result suggests that the performance of the protocol is improved if the average number of tags participating in each query is low (i.e., $N<20$ ).

\section{Proposed Algorithm For FAst IDENTIFICATION}

In this section, we describe the proposed mechanism for fast identification and we compare the performance with the standard ISO/IEC 18000-6C MAC.

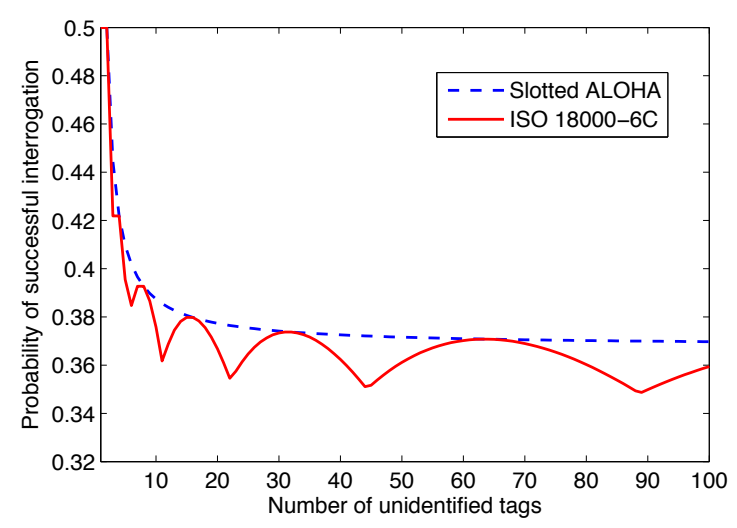

Fig. 3. Probability of successful interrogation vs. current number of unidentified tags.

\section{A. Protocol description}

The ISO/IEC 18000-6C MAC is designed by considering that the number of tags and their position are not known a priori. However, in the presence of UWB modules, the reader can perform accurate and fast ranging in parallel with the ISO/IEC 18000-6C MAC. Then, it is possible to adapt the transmission range of the reader so that the average number of tags in each query is kept under control. The proposed integration works as follows:

\section{$U H F$ power up and $U W B$ ranging}

- The reader transmits a continuos-wave UHF signal to power up the tags in its maximum transmission range RangeMax.

- The reader sends a sequence of UWB pulses and tags respond by modulating the backscattered UWB signal.

- The reader estimates the number of tags and the relative distance by considering the time of arrival (ToA) of the backscattered signals.

\section{UHF interrogation}

- The reader sends a Query command with sufficient power to interrogate $N_{0}$ tags (Range 0 ), and selecting $Q=\left[\log _{2} N_{0}\right]$. where $[\cdot]$ denotes the nearest integer function

- After $x \leq N_{0}$ tags have been identified, the reader increases the transmission power of the following Query command to reach and include $x$ further tags to the interrogation (Range1).

- The transmission range is increased every $x$ successful interrogations until it reaches RangeMax.

- Between consecutive Query commands, the interrogation proceeds by using QueryRep and Query Adj commands according to the standard procedure in Sect. IV. The transmission power is set to reach also tags that are interrogated in the next Query. By doing so, those tags are already powered when receiving the Query command.

- The session ends when all tags have been identified.

We recall that the proposed adaptation is compliant with the ISO/IEC 18000-6C specifications for both the reader and the tag. The optimal choice of the parameters $N_{0}$ and $x$ is not 


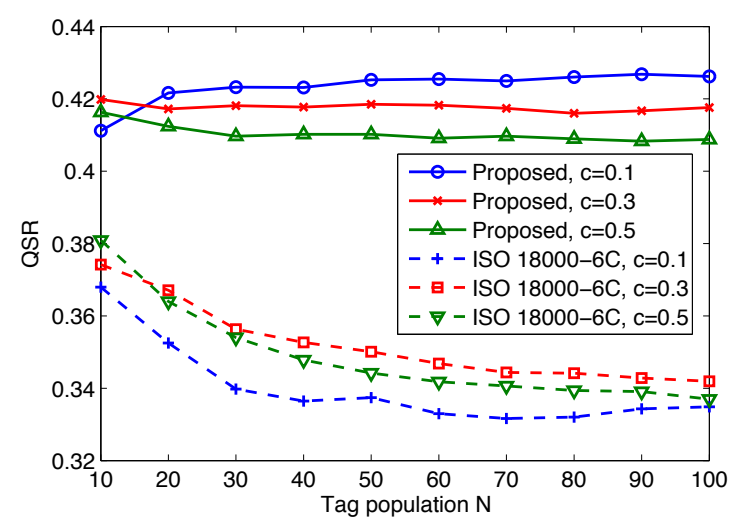

Fig. 4. QSR vs. tag population. The proposed approach guarantees $25 \%$ improvement to the standard ISO/IEC 18000-6C for large-scale networks.

straightforward. Every time the reader includes new tags, it has to issue a Query command instead of a shorter QueryAdj command. Therefore, if $N_{0}$ and $x$ are too small, the TIS is negatively affected.

\section{B. Performance results}

A single reader is deployed to interrogate a population of $N=[10-100]$ tags operating with the standard ISO/IEC 18000-6C. The air interface parameters are set to default specifications in [4] for tag modulation rate of $125 \mathrm{KHz}$.

We show the performance results in terms of QSR in Fig. 4 and TIS in Fig. 5 of the standard ISO/IEC 18000-6C MAC and the proposed approach with $N_{0}=10$ and $x=5$ for various values of $c$. We recall that a larger value of $c$ indicates fast adaptation of the backoff window after collided and idle rounds (see Sect. IV). The proposed mechanism improves the performance of the ISO/IEC 18000-6C MAC in the considered range of tag population and values of $c$. For $c=0.1$ and $N=100$, the proposed mechanism achieves $\mathrm{QSR} \approx 0.43$ with $25 \%$ gain with respect to the ISO/IEC 18000-6C, and TIS $=513 \mathrm{tags} / \mathrm{s}$ with $7 \%$ gain in terms of tag identification speed as the tag population increases.

\section{CONClusions And Future Work}

System architectures and protocol mechanisms for ISO/IEC 18000-6C systems that integrate UWB technology have been investigated in this paper. We have proposed an identification mechanism that uses UWB ranging information to improve the speed of identification. Protocol performance have been validated by means of simulations in various scenarios. As a future work, we aim at deriving optimal parameters $N_{0}$ and $x$, depending on the ranging accuracy. Moreover, we plan to implement the proposed architecture and the protocol enhancements on ISO/IEC 18000-6C compliant hardware.

\section{REFERENCES}

[1] R. Das and P. Harrop, "RFID forecast, players and opportunities 20072017," http://www.idtechex.com.

[2] D. Dardari, R. D'Errico, C. Roblin, A. Sibille, and M. Z. Win, "Ultrawide bandwidth RFID: The next generation?" Proceedings of the IEEE, Special Issue on RFID - A Unique Radio Innovation for the 21st Century., vol. 98, no. 9, pp. $1570-1582$, Sep 2010.

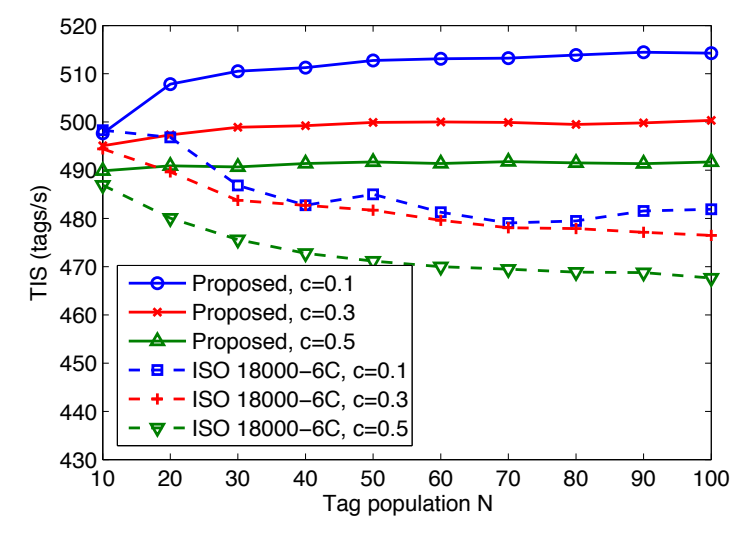

Fig. 5. TIS vs tag population. The proposed approach guarantees 7\% improvement to the standard ISO/IEC 18000-6C for large-scale networks.

[3] D. Dardari, A. Conti, U. Ferner, A. Giorgetti, and M. Z. Win, "Ranging with ultrawide bandwidth signals in multipath environments," Proceedings of the IEEE, Special Issue on UWB Technology \& Emerging Applications, vol. 97, no. 2, pp. 404-426, Feb 2009.

[4] ISO/IEC 18000 - Radio frequency identification for item management - Part 6: Parameters for air interface communications at $860 \mathrm{MHz}$ to $960 \mathrm{MHz}, 2013$.

[5] EPCGlobal, Class 1 Generation 2 UHF Air interface protocol standard, 2005.

[6] Y.-C. Ko, S. Roy, J. Smith, H.-W. Lee, and C.-H. Cho, "RFID MAC performance evaluation based on ISO/IEC 18000-6 type C," IEEE Communications Letters, vol. 12, no. 6, pp. 426-428, 2008.

[7] V. Namboodiri and L. Gao, "Energy-aware tag anticollision protocols for RFID systems," IEEE Transactions on Mobile Computing, vol. 9 , no. 1, pp. 44-59, 2010.

[8] Y. Cui and Y. Zhao, "Performance evaluation of a multi-branch tree algorithm in RFID," IEEE Transactions on Communications, vol. 58, no. 5, pp. 1356-1364, 2010.

[9] L. Chen, I. Demirkol, and W. Heinzelman, "Token-MAC: A fair MAC protocol for passive RFID systems," in IEEE Global Telecommunications Conference (GLOBECOM), 2011.

[10] C. Wang, M. Daneshmand, K. Sohraby, and B. Li, "Performance analysis of RFID generation-2 protocol," IEEE Transactions on Wireless Communications, vol. 8, no. 5, pp. 2592-2601, 2009.

[11] F. Hessar and S. Roy, "Energy based performance evaluation of passive EPC Gen 2 Class 1 RFID systems," IEEE Transactions on Communications, vol. 61, no. 4, pp. 1337-1348, 2013.

[12] D. De Donno, L. Tarricone, V. Lakafosis, and M. Tentzeris, "Multipacket reception MAC schemes for the RFID EPC Gen2 protocol," in International Symposium on Wireless Communication Systems (ISWCS), 2012, pp. 311-315.

[13] L. Zhu and T. Yum, "Optimal framed aloha based anti-collision algorithms for RFID systems," IEEE Transactions on Communications, vol. 58, no. 12, pp. 3583-3592, 2010.

[14] Y. Maguire and R. Pappu, "An optimal q-algorithm for the ISO 180006C RFID protocol," IEEE Transactions on Automation Science and Engineering, vol. 6, no. 1, pp. 16-24, 2009.

[15] P. Di Marco, F. Santucci, and C. Fischione, "Modeling anti-collision protocols for RFID systems with multiple access interference," in IEEE International Conference on Communications (ICC), 2014.

[16] D. Dardari and R. D'Errico, "Passive ultrawide bandwidth rfid," in IEEE Global Telecommunications Conference (GLOBECOM), 2008.

[17] M. Z. Win, D. Dardari, A. F. Molisch, W. Wiesbeck, and Z. Jinyun, "History and applications of UWB," Proceedings of the IEEE, Special Issue on UWB Technology \& Emerging Applications, vol. 97, no. 2, pp. 198-204, Feb 2009.

[18] C. Xu and C. L. Law, "TOA estimator for UWB backscattering RFID system with clutter suppression capability," EURASIP Journal on Wireless Communication Networks, pp. 46:1-46:13, Apr. 2010.

[19] M. Mohaisen, H. Yoon, and K. Chang, "Radio transmission performance of EPCglobal Gen-2 RFID system," in International Conference on Advanced Communication Technology, vol. 2, 2008, pp. 1423-1428.

[20] R. Rom and M. Sidi, Multiple Access Protocols: Performance And Analysis. Springer-Verlag. New York., 1990. 